\title{
Inner Spiral Arms of the Barred Galaxy NGC 4314
}

\author{
H. B. Ann ${ }^{1}$ and K. H. Kwon \\ Department of Earth Sciences, Pusan National University, Pusan, \\ 609-735, Korea
}

\begin{abstract}
The nuclear region of the barred galaxy NGC 4314 was observed and modeled by $V I J H K$ photometry, long-slit spectroscopy, and SPH simulations. Young stellar populations is supposed to be made from the gas which are accumulated in the nuclear ring and/or inner spiral arms by the strong bar potential.
\end{abstract}

\section{Introduction}

The barred spiral galaxy NGC 4314 has been known to have a complicated internal structure which implies an ongoing dynamical evolution in the nuclear region of the galaxy. Detailed morphology and optical/near IR photometry were given by Benedict et al. (1992), and CO, HI and radio continuum along with optical and near IR observations were made by Garcia-Barreto et al. (1991). These observations show that NGC 4314 has inner spiral arms which emerge from the nuclear ring whose diameter is about $500 \mathrm{pc}$ and there is little HI over the entire galaxy. Recent HST images of the nuclear region of NGC 4314 (Benedict et al. 1993) clearly shows that the nuclear ring is a nuclear spiral which is delineated by the dust distribution.

It is well known that a nuclear ring can be formed at ILR due to the bar potential. However, detailed morphology of the nuclear rings depends on the distribution of masses in distinct components as well as bar strength. Since NGC 4314 seems to be a classic example of a barred galaxy whose bar drives disk gas into a ring at ILR, it is important to analyze the inner structure of NGC 4314 in detail, in terms of morphology and dynamics. We conducted VIJHK surface photometry and long-slit spectroscopy to investigate the morphology and stellar content of the nuclear region of NGC 4314. The dynamical properties of the central region of NGC 4314 were modeled by SPH simulations.

\section{Observations}

We obtained direct images of NGC 4314 using a $1024 \times 1024$ Tek CCD with Johnson-Cousins $V$ and $I$ filters, and $256 \times 256$ IR arrays with $J, H$, and $K$ filters on the $1.8 \mathrm{~m}$ telescope of the Dominion Astrophysical observatory (DAO).

\footnotetext{
${ }^{1}$ Visiting Astronomer, Dominion Astrophysical Observatory.
} 
The pixel scale was 0.47 arcsec per pixel for the Tek CCD and 0.67 arcsec per pixel for the IR arrays.

Two sets of long-slit spectra of the nuclear region of NGC 4314 were obtained with the Cassegrain spectrograph mounted on the DAO $1.8 \mathrm{~m}$ telescope. In one setting the grating gave a coverage from $4750 \AA$ to $5700 \AA$ at $8.0 \AA$ resolution. The other setting covered the wavelength region $6100 \AA$ to $6750 \AA$ with $4.0 \AA$ resolution. The data reduction followed standard procedures using IRAF.

\section{Morphology and Stellar Content}

The bulge of NGC 4314 seems to be small compared with that of other earlytype spiral galaxies. There are isophotal twists which suggest that the bulge of NGC 4314 is triaxial. The bar is very prominent with an axial ratio of $\sim 3$. The luminosity of the bar dominates the galaxy luminosity outside the nuclear bulge. The inner spiral arms can be seen in the $V$ - and $I$-band images. But there is no apparent signature of spiral patterns in $J H K$ images.

The nuclear ring and/or inner spiral arms are made of hot spots and dust patches. The existence of young stellar populations in the nuclear ring is also suggested by the long-slit spectra. There is strong $\mathrm{H} \alpha$ and $\mathrm{H} \beta$ emission from the nuclear ring, but virtually no $\mathrm{H} \alpha$ and $\mathrm{H} \beta$ emission from the nucleus itself. This suggests that recent star formation is confined to the nuclear ring and/or inner spiral arms.

\section{SPH Simulations}

We applied the SPH method to simulate the formation and evolution of the nuclear ring of NGC 4314. We assumed an exponential disk (Freeman 1970) and Plummer spherical potential (Plummer 1911)

$$
\Phi(r)=-\frac{G M}{\sqrt{\left(r^{2}+b^{2}\right)}},
$$

for bulge components. For the bar component, we used the bi-axial bar potential of Long \& Murali (1992). The bar is assumed to be homogeneous and constant in time. Gas particles are uniformly distributed at the beginning of the simulations and assumed to be isothermal with a temperature of $10,000 \mathrm{~K}$. The models are confined to the radius of $4 \mathrm{kpc}$ with a total mass of $2 \times 10^{10} M_{\odot}$. We assumed the mass and axial ratio of the bar to be $0.3 M_{\text {tot }}$ and 3 , respectively. We tried several models of different bulge to disk mass ratios.

In our models with an ILR, gas inside CR flows to the ILR to make a nuclear ring. But there is little gas in the nucleus itself. Gas inflow is effective when the bulge is massive and centrally concentrated. For the large bulge models with $\frac{M_{\text {bulge }}}{M_{\text {disk }}} \sim 1.2,1.5 \times 10^{7} M_{\odot}$ of gas is accumulated to the inner $400 \mathrm{pc}$ and $1 \times 10^{8} M_{\odot}$ of gas is accumulated to the inner $1.3 \mathrm{kpc}$ within $3 \times 10^{8} \mathrm{yrs}$ if the initial mass of the uniform gas disk is $2 \times 10^{8} M_{\odot}$. In this model, $0.5 M_{\odot} y r^{-1}$ of stars can be formed in the nuclear ring for the period of $3 \times 10^{8} \mathrm{yrs}$.

The orientation of the nuclear ring is sensitive to the bulge to disk mass ratio and bulge size. A massive and/or concentrated bulge allows an ILR with high 


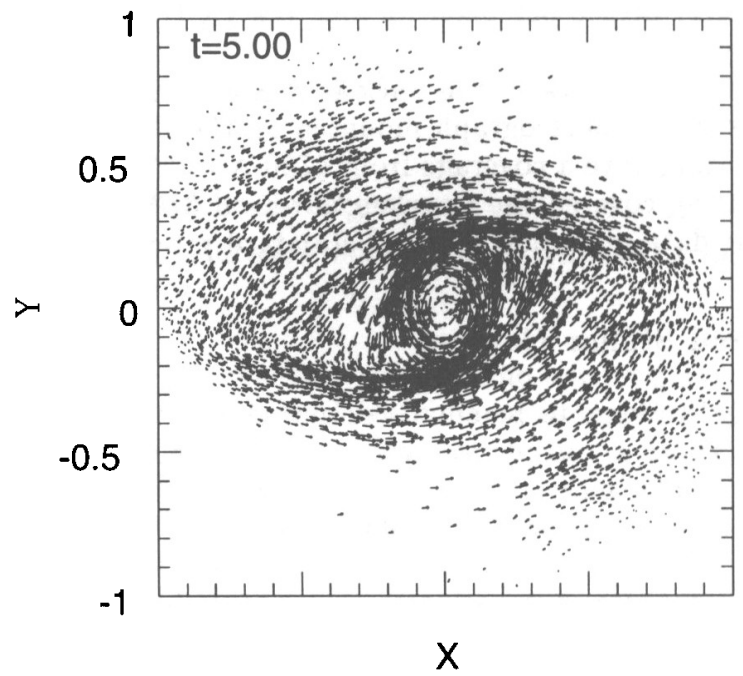

Figure 1. Gas velocity at $t=5 \times \tau_{s c}$, where $\tau_{s c}=(G \bar{\rho})^{-\frac{1}{2}}$, for a massive bulge model having $\frac{M_{b u l g e}}{M_{\text {disk }}} \sim 1.2$. Shock fronts are developed into a two-arm spiral pattern.

pattern speed and makes the nuclear ring elongated perpendicular to the bar. For the small bulge models, the nuclear ring is formed with random orientation, but in later times it becomes aligned with the bar. Figure 1 shows a snapshot of gas motions for the model with $\frac{M_{\text {bulge }}}{M_{\text {disk }}} \sim 1.2$, just after the formation of a nuclear ring at ILR. The nuclear ring appears to be elongated perpendicular to the bar. The velocity of gas particles clearly shows the shock fronts of spiral pattern.

Acknowledgments. We are grateful to Dr. Hyesung Kang and Hyung Mok Lee for many discussions. This work was supported in part by the Basic Science Research Program, Ministry of Education.

\section{References}

Benedict, G. F., Higdon, J. L., Tollestrup, E. V., Hahn, J., \& Harvey, P. M. 1992, AJ, 103, 757

Benedict, G. F., Higdon, J. L., Jefferys, W. H., Duncombe, R., Hemenway, P. D., Shelus, P. J., Whipple, A. L. et al. 1993, AJ, 105, 1369

Freeman, K. C. 1970, ApJ, 160, 811

Garcia-Barreto, J. A., Downes, D., Combes, F., Gerin, M., Megri, C., Carrasco, L., \& Cruz-Gonzalez, I. 1991, A\&A, 244, 257

Long, K. \& Murali, C. 1992, ApJ, 397, 44

Plummer, H. C. 1911, MNRAS, 71, 460 